\title{
Ion-Conserving Modified Poisson-Boltzmann Theory Considering a Steric Effect in an Electrolyte
}

\author{
Hideyuki Sugioka* \\ Department of Mechanical Systems Engineering, \\ Shinshu University, 4-17-1 Wakasato, Nagano 380-8553, Japan
}

(Dated: October 28, 2016)

\begin{abstract}
The modified Poisson-Nernst-Planck (MPNP) and modified Poisson-Boltzmann (MPB) equations are well known as fundamental equations that consider a steric effect, which prevents unphysical ion concentrations. However, it is unclear whether they are equivalent or not. To clarify this problem, we propose an improved free energy formulation that considers a steric limit with an ion-conserving condition and successfully derive the ion-conserving modified Poisson-Boltzmann (IC-MPB) equations that are equivalent to the MPNP equations. Furthermore, we numerically examine the equivalence by comparing between the IC-PB solutions obtained by the Newton method and the steady MPNP solutions obtained by the finite-element finite-volume method. A surprising aspect of our finding is that the MPB solutions are much different from the MPNP (IC-MPB) solutions in a confined space. We consider that our findings will significantly contribute to understanding the surface science between solids and liquids.
\end{abstract}

PACS numbers:

*Electronic address: hsugioka@shinshu-u.ac.jp 


\section{INTRODUCTION}

The steric effect of ions in an electrolyte is important because it prevents the unphysical concentration of ions when we consider the behaviors of ions in a crowding state; e.g., it can reasonably correct the unphysical solutions [1-5] of the well-known Gouy-Chapman (GC) model of the diffused layer near electrodes at high applied voltages $(>25 \mathrm{mV})[6,7]$. Note that the GC solution provides a $10^{40}$ times higher concentration than the initial bulk ion concentration near the electrode of $1 \mathrm{~V}$; it is of course unphysical, as pointed out by many researchers [1-5], although Grahame [8] successfully explained the experimental results of the differential capacity by using the GC model along with the Stern layer model. In addition, the ion crowding state can be found near general polarizable objects not only in a dense electrolyte but also in a very dilute electrolyte at high applied voltages, and it may cause a significant decrease in the device performance using electrokinetic phenomena [5]. Hence, many studies have been devoted to this problem [1-5]. In particular, Borukhov et al. proposed the modified Poisson-Boltzmann (MPB) equation based on a free energy formulation considering a steric effect [1]. Kilic et al. proposed the modified Poisson-Boltzmann (MPB) equation based on the free energy formulation with chemical potential arguments [3]. Obviously, the MPNP and MPB equations are important milestones that provide an integrated viewpoint from low to high voltage phenomena and from dilute to dense phenomena in electrolyte dynamics. However, it is unclear whether they provide the same solution or not, although we consider that they should provide the same solution for the same physical problem if they are true fundamental equations. To overcome the difficulty, we propose an improved free energy formulation considering a steric effect with an ion-conserving condition because we already showed that the classical PB equation becomes equivalent to the the classical PNP equations by adding an ion-conserving condition [9]. In addition, this kind of fundamental theory is also required to improve promising microfluidic devices using electroosmosis, as discussed in Ref. [10]. Thus, on the basis of the new thermodynamic formulation, we derive a new MPB equation, i.e., the ion-conserving Poisson-Boltzmann (IC-MPB) equations, which are equivalent to the steady MPNP equations that consider a steric effect. Namely, in this study, we focus on deriving the IC-MPB equation equivalent to the steady MPNP equations and numerically examine the consistency. Note that for readers' convenience, in Table I, we show a list of the symbols and abbreviations used. 
TABLE I: List of symbols and abbreviations.

\begin{tabular}{l|l}
\hline Symbol & Property \\
\hline \hline GC & Gouy-Chapman \\
PB & Poisson-Boltzmann \\
MPB & Modified PB \\
ICPB & Ion-Conserving PB \\
IC-MPB & Ion-Conserving MPB \\
PNP & Poisson-Nernst-Planck \\
MPNP & Modified MPNP \\
$F_{h}$ & modified total Helmholtz free energy \\
$\Phi(\phi)$ & dimensional (nondimensional) electrostatic potential \\
$\lambda$ & Lagrange multiplier \\
$V_{0}\left(v_{0}\right)$ & dimensional (nondimensional) applied voltage \\
$C_{ \pm}$ & positive and negative ion concentrations \\
$C_{0}$ & initial bulk ion concentration \\
$C_{b}$ & bulk ion concentration at $V_{0}$ \\
$u$ & internal energy per unit volume \\
$s$ & entropy per unit volume \\
$I_{0}$ & initial ion number \\
$a$ & ion size (approximately, ion diameter) \\
$\lambda_{D}$ & ion charge \\
$\varepsilon$ & Debye screening length \\
\hline$C_{0} a^{3}$ (nondimensional value) \\
\hline \begin{tabular}{l}
$W_{0} / \lambda_{D}$ (nondimensional value) \\
\hline
\end{tabular}
\end{tabular}




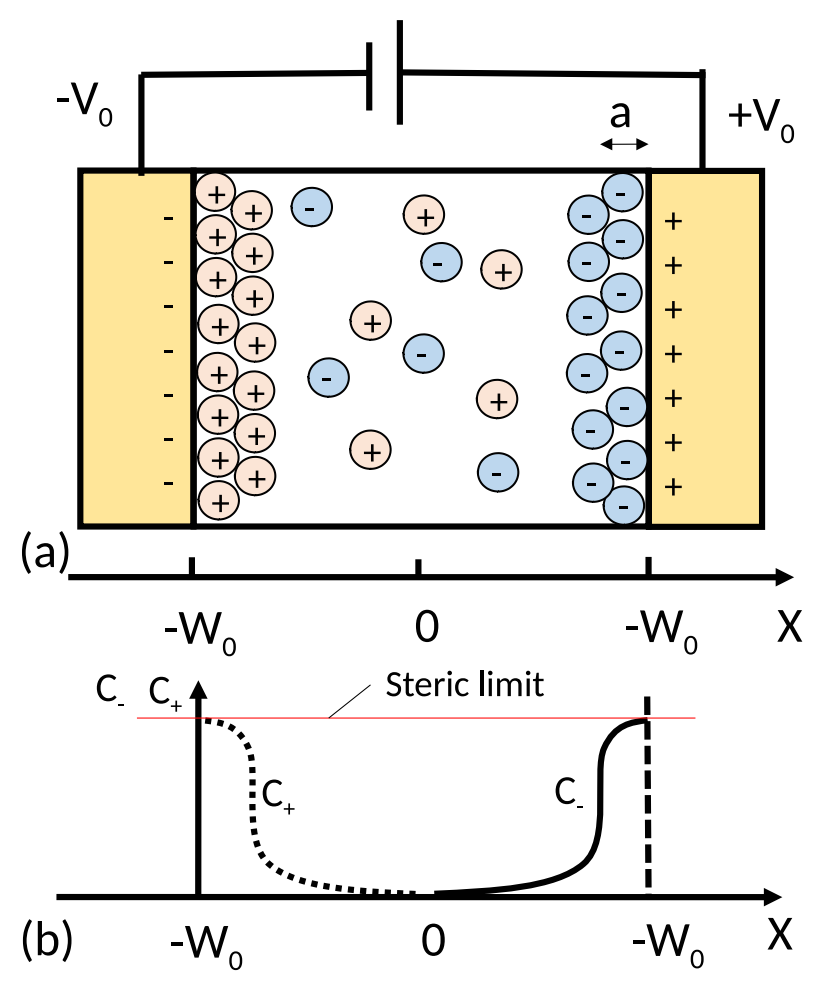

FIG. 1: (Color online) Electrolyte-electrode system used in our 1D examination. Note that we examine our theory in this 1D system, although our IC-MPB theory is considered for 3D systems. Here, $2 V_{0}$ is an applied voltage and $2 W_{0}$ (typically, $W_{0}=100 \mu \mathrm{m}$ ) is the distance between the electrodes.

\section{THEORY}

\section{A. Improved total free energy formulation considering a steric effect with an ion-} conserving condition

The fundamental assumption of the improved total free energy for the MPNP and ICMPB theories is almost the same as that of the conventional MPB and MPNP theories [1-5] except that it includes an ion-conserving condition. However, since the difference is essential, we explain it from the beginning. That is, within the framework of mean-field approximation, the modified total Helmholtz free energy $F_{h}$ that considers steric effects with the ion-conserving condition can be written as the functional of the electrostatic potential 
$\Phi(\boldsymbol{r})$, the derivative $\nabla \Phi(\boldsymbol{r})$, the positive and negative ion concentrations (i.e., cation and anion concentrations) $C_{ \pm}(\boldsymbol{r})$, and the Lagrange multiplier $\lambda$ for the ion-conserving condition. Namely, by considering the ion-conserving condition along with the treatment of Borukohov et al. [1], we can write $F_{h}$ as

$$
F_{h}=F_{h}\left(\Phi, \nabla \Phi, C_{+}, C_{-}, \lambda\right)=\int(u-T s) d \boldsymbol{r}+\lambda\left[\int\left(C_{+}+C_{-}\right) d \boldsymbol{r}-I_{0}\right]
$$

where $u, s$, and $f(\equiv u-T s)$ are the internal energy, entropy, and Helmholtz free energy, respectively, for a unit volume, $T$ is the absolute temperature, and $I_{0}$ is the initial ion number. Note that the second term of Eq. (1) is the constraint due to the ion-conserving condition and the assumption is very natural as a fundamental assumption because the mass conservation is the most fundamental law of nature. Furthermore, the specific internal energy due to the self-energy of the electric field $\left(-\frac{\varepsilon}{2}|\nabla \Psi|^{2}\right)$ and the electrostatic energies of ions is denoted as

$$
u=-\frac{\varepsilon}{2}|\nabla \Phi|^{2}+z e C_{+} \Phi-z e C_{-} \Phi
$$

the entropic contribution is denoted as

$$
-T s=\frac{k T}{a^{3}}\left[\ln \left(C_{+} a^{3}\right)^{\left(C_{+} a^{3}\right)}+\ln \left(C_{-} a^{3}\right)^{\left(C_{-} a^{3}\right)}+\ln \left(1-C_{+} a^{3}-C_{-} a^{3}\right)^{\left(1-C_{+} a^{3}-C_{-} a^{3}\right)}\right],
$$

and the initial ion number $I_{0}$ is denoted as

$$
I_{0}=\int\left(C_{0}+C_{0}\right) d \boldsymbol{r}
$$

where $C_{0}$ is the initial bulk ion concentration in the absence of electric fields and $k$ is the Boltzmann constant. Similar to the treatment of Refs. [1-5], we assume that the concerned ion system is a symmetrical z:z electrolyte system of the ion charge ze; for simplicity, the concerned positive and negative ions and the solvent ions are considered to have the same size $a$. Here, $a$ is approximately the diameter of ions as the first approximation, although it is rigorously defined as $a \equiv\left(\frac{V_{c}}{N_{c}}\right)^{\frac{1}{3}}$, where $V_{c}$ is the volume of a condensed state and $N_{c}$ is the total number of ions (of the state) including positive and negative ions. Furthermore, the last term of Eq. (3) is the most important term for steric effects and it shows a significant increase in entropy when $\left(C_{+}+C_{-}\right) a^{3}$ approaches one. 


\section{B. Derivation of ion-conserving modified Poisson-Boltzmann (IC-MPB) equa-} tions

On the basis of the improved total free energy formulation of Eq. (1), we can develop the IC-MPB theory that is a natural extension from the conventional MPB theory $[1,3]$ and the ion-conserving Poisson-Boltzmann (IC-PB) theory [9]. Namely, by considering Eular's equation for the functional derivatives, the functional derivatives of $F_{h}$ in Eq. (1) concerning $\Psi, C_{ \pm}$, and $\lambda$ are described as

$$
\begin{array}{r}
\frac{\delta F_{h}}{\delta \Phi} \equiv \frac{\partial f}{\partial \Phi}-\nabla\left(\frac{\partial f}{\partial(\nabla \Phi)}\right)=e z\left(C_{+}-C_{-}\right)+\varepsilon \nabla^{2} \Phi \\
\frac{\delta F_{h}}{\delta C_{ \pm}} \equiv \frac{\partial f}{\partial C_{ \pm}}= \pm e z \Phi+k T \ln \frac{C_{ \pm} a^{3}}{\left(1-C_{+} a^{3}-C_{-} a^{3}\right)} \\
\frac{\delta F_{h}}{\delta \lambda} \equiv \frac{\partial F_{h}}{\partial \lambda}=\int\left(C_{+}+C_{-}-2 C_{0}\right) d \boldsymbol{r}
\end{array}
$$

Thus, by requiring $\frac{\delta F_{h}}{\delta \Phi}=0$, we obtain the following Poisson equation as the equation that provides the stational point for $F$ : i.e.,

$$
-\varepsilon \nabla^{2} \Phi=e z\left(C_{+}-C_{-}\right)
$$

Similarly, by requiring $\mu=\frac{\delta F_{h}}{\delta C_{ \pm}}=$const. (a constant chemical potential condition for a steady state) and $\frac{\delta F_{h}}{\delta \lambda}=0$ (the ion-conserving condition), we obtain the IC-MPB equations as

$$
\begin{array}{r}
\nabla^{2} \Phi=\frac{2 z e C_{b}}{\varepsilon} \frac{\sinh \frac{e z \Phi}{k T}}{1+2 C_{b} a^{3} \cosh \frac{e z \Phi}{k T}}, \\
\int\left(C_{+}+C_{-}-2 C_{0}\right) d \boldsymbol{r}=0,
\end{array}
$$

where $C_{b}$ is determined by the ion-conserving condition of Eq. (10), and the physical meaning of $C_{b}$ is the bulk concentration of the steady state under the existence of the applied voltage $V_{0}$. Note that, in the conventional MPB theory, the value of $C_{b}$ is usually selected as $C_{b}=C_{0}$ without special arguments despite the arbitrariness. However, in microfluidic configurations, the selection becomes unphysical from the viewpoint of the ion-conserving condition of Eq. (10), as will be explained later. Namely, the solution of Eq. (9) at $C_{b} / C_{0}=1$ is the solution of the conventional MPB method, whereas the solution of Eq. (9) at the value of $C_{b} / C_{0}$ that satisfies Eq. (10) is the solution of the IC-MPB method. Furthermore, note that the condition $\mu=\frac{\delta F_{h}}{\delta C_{ \pm}}=$const. provides Eq. (9) with the arbitrary parameter of $C_{b}$ 
and Eq. (9) provides a group of solutions (i.e., the solutions of the general PB equation) by setting the value of $C_{b}$ arbitrarily.

Although the process of deriving Eqs. (9) and (10) is almost the same as that of the conventional MPB and MPNP equations, we explain the process briefly to clarify the difference among them. Namely, $\frac{\delta F_{h}}{\delta C_{ \pm}}$corresponds to the chemical potential $\left(\mu^{\text {steric }}\right)$ that considers steric effects. However, physically, we can choose the standard point for measuring $\mu^{\text {steric }}$ and only the difference in $\mu^{\text {steric }}$ has a physical meaning. Thus, we can define the chemical potential as

$$
\mu^{\text {steric }, \alpha} \equiv \frac{\delta F_{h}}{\delta C_{ \pm}}+\alpha
$$

where $\alpha$ is an arbitrary constant and the value is selected conveniently. For example, by setting $\alpha=-k T \ln a^{3}$, we can remove the constant term $\left(k T \ln a^{3}\right)$ in Eq. (6) and we obtain the usual form of $\mu^{\text {steric }}$ as $\mu^{\text {steric,usual }}= \pm e z \Phi+k T \ln \frac{C_{ \pm}}{\left(1-C_{+} a^{3}-C_{-} a^{3}\right)}$. Note that Kilic et al. derived their MPNP equations using the formulation with the continuous equation. By using the other arbitrary constant $\beta$, we can also write the chemical potential as

$$
\mu^{\text {steric }, \beta}= \pm e z \Phi+k T \ln \frac{C_{ \pm}\left(\frac{1}{\beta}\right)}{\left(1-C_{+} a^{3}-C_{-} a^{3}\right)} .
$$

This argument is important for the IC-MPB theory since the breaking of the ion-conserving condition of the conventional MPB theory results from the arbitrariness of the $\alpha$ or $\beta$ value in the chemical potential. In fact, in the conventional MPB theory, by setting that $\beta=C_{0}$ and requiring $\mu^{\text {steric }, \beta}=0$, one usually obtains the ion concentration $C_{ \pm}^{\text {conv. }}=$ $C_{0}\left(1-C_{+} a^{3}-C_{-} a^{3}\right) \mathrm{e}^{\mp \frac{e z \Phi}{k T}}$, where $C_{0}$ is the ion concentration of the bulk in the absence of an electric field. However, this solution does not ensure the ion-conserving condition. Therefore, in our IC-MPB theory, by setting $\beta=C_{b}$ and requiring $\mu^{\text {steric, } \beta}=0$, we obtain the ion concentration for the IC-MPB theory as

$$
C_{ \pm}=C_{b}\left[1-\left(C_{+}+C_{-}\right) a^{3}\right] \mathrm{e}^{\mp \frac{e z \Phi}{k T}}
$$

where $C_{b}$ is the modified bulk concentration that satisfies the ion-conserving condition

$$
\int\left(C_{+}+C_{-}\right) d \boldsymbol{r}=\int 2 C_{0} d \boldsymbol{r}
$$

Furthermore, by using Eq. (13), we obtain $C_{+}+C_{+}=2 C_{b}\left[1-\left(C_{+}+C_{-}\right) a^{3}\right] \cosh \frac{e z \Phi}{k T}$, and thus

$$
C_{+}+C_{-}=\frac{2 C_{b} \cosh \frac{e z \Phi}{k T}}{1+2 C_{b} a^{3} \cosh \frac{e z \Phi}{k T}} .
$$


Therefore, the ion-conserving condition is converted into

$$
\int \frac{2 C_{b} \cosh \frac{e z \Phi}{k T}}{1+2 C_{b} a^{3} \cosh \frac{e z \Phi}{k T}} d \boldsymbol{r}=\int 2 C_{0} d \boldsymbol{r} .
$$

Furthermore, by using Eq. (13), we obtain $C_{+}-C_{+}=-2 C_{b}\left[1-\left(C_{+}+C_{-}\right) a^{3}\right] \sinh \frac{e z \Phi}{k T}$, and thus $C_{+}-C_{-}=-2 C_{b}\left[1-\frac{2 C_{b} \cosh \frac{e z \Phi}{k T}}{1+2 C_{b} a^{3} \cosh \frac{e z \Phi}{k T}}\right] \sinh \frac{e z \Phi}{k T}$; i.e., we obtain

$$
C_{+}-C_{-}=\frac{-2 C_{b} \sinh \frac{e z \Phi}{k T}}{1+2 C_{b} a^{3} \cosh \frac{e z \Phi}{k T}} .
$$

By substituting Eq. (13) into the Poisson equation [Eq. (8)], we obtain one of the IC-MPB equations as

$$
\nabla^{2} \Phi=\frac{2 z e C_{b}}{\varepsilon} \frac{\sinh \frac{e z \Phi}{k T}}{1+2 C_{b} a^{3} \cosh \frac{e z \Phi}{k T}}
$$

with the ion-conserving condition of Eq. (16). Furthermore, from Eqs. (15) and (17), we obtain

$$
C_{ \pm}=\frac{C_{b} \mathrm{e}^{\mp \frac{e z \Phi}{k T}}}{1+2 C_{b} a^{3} \cosh \frac{e z \Phi}{k T}} .
$$

For readers' convenience, we only summarize the nondimensional form of the IC-MPB equations as

$$
\begin{array}{r}
\nabla^{2} \phi=\frac{c_{b} \kappa^{2} \sinh \phi}{1+c_{b} \nu \cosh \phi} \\
\int \frac{2 c_{b} \cosh \phi}{1+c_{b} \cosh \phi} d \boldsymbol{r}_{n}=\int 2 d \boldsymbol{r}_{n}, \\
c_{ \pm}=\frac{c_{b} \mathrm{e}^{\mp \phi}}{1+c_{b} \nu \cosh \phi}
\end{array}
$$

where $2 C_{0} a^{3}=\nu, c_{b}=C_{b} / C_{0}, c_{ \pm}=C_{ \pm} / C_{0}, \phi=\frac{e z \Phi}{k T}=\Phi / \Phi_{c}, \Phi_{c}=\frac{k T}{e z}, \kappa=W / \lambda_{D}$, $\boldsymbol{r}_{n}=\boldsymbol{r} / W, W$ is a characteristic length, and $\lambda_{D}$ is the Debye screening length.

\section{Derivation of the steady MPNP equations from the IC-MPB equations}

Kilic et al. derived the MPNP equations by using the phenomenological argument with the chemical potential $\mu^{\text {steric,usual }}$ [4]. Thus, it is unclear whether the steady MPNP equation is equivalent to the IC-MPB equations or not. Therefore, to clarify the problem, we here consider the spatial derivative of $C_{ \pm}$in Eq. (13) as

$$
\nabla C_{ \pm}=\mp \frac{e z}{k T} C_{b}\left[1-\left(C_{+}+C_{-}\right) a^{3}\right] \mathrm{e}^{\mp \frac{e z \Phi}{k T}} \nabla \Phi-a^{3} C_{b}\left[\nabla\left(C_{+}+C_{-}\right)\right] \mathrm{e}^{\mp \frac{e z \Phi}{k T}} .
$$


Thus, by using Eq. (13) and its converted formulation $C_{b} \mathrm{e}^{\mp \frac{e z \Phi}{k T}}=C_{ \pm} /\left[1-\left(C_{+}+C_{-}\right) a^{3}\right]$, we obtain

$$
\nabla C_{ \pm}=\mp \frac{e z}{k T} C_{ \pm} \nabla \Phi-\frac{a^{3} C_{ \pm}}{\left[1-\left(C_{+}+C_{-}\right) a^{3}\right]} \nabla\left(C_{+}+C_{-}\right)
$$

Note that the parameter of $C_{b}$ does not appear in Fig. (24) and the ion-conserving condition of the MPNP equation is ensured by using the continuous equations with the initial conditions. Thus, we find that the IC-MPB equations proposed by us and the MPNP equations proposed by Kilic et al. [4] are theoretically equivalent to each other for the steady state, and that both equations are justified as fundamental equations, whereas the conventional MPB equation might not be suitable at high applied voltages because it lacks the ion-conserving condition. Note that the non-dimensional form of Eq. (24) is

$$
\nabla c_{ \pm}=\mp c_{ \pm} \nabla_{n} \phi-\frac{\nu c_{ \pm}}{\left[2-\nu\left(c_{+}+c_{-}\right)\right]} \nabla_{n}\left(c_{+}+c_{-}\right)
$$

where $\nabla_{n}$ is a nondimensional operator corresponding to $\nabla$.

\section{Electrolyte-electrode system used in our 1D examination}

Figure 1 shows an electrolyte-electrode system used in our 1D examination; i.e., we examine our theory in this 1D system, although our IC-MPB theory can be used in 2D and $3 \mathrm{D}$ systems. In Fig. 1, $2 V_{0}$ is an applied voltage and $2 W_{0}$ (typically, $100 \mu \mathrm{m}$ ) is the distance between the electrodes. As shown in Fig. 1, by applying voltages between electrodes, negative and positive ions move to the positive and negative electrodes, respectively. In particular, at high applied voltages $(>25 \mathrm{mV})$, an ion crowding state can be seen, and owing to the steric effect, the condensation layer can be formed near electrodes, as pointed out by many researchers [1-5]. However, in a confined space, the ion-conserving condition also becomes important along with the steric effect, and the complex phenomena often should be described for the innovative microfluidic device designs. Thus, our IC-MPB theory becomes important particularly in the microfluidic systems.

\section{E. Newton method for solving the IC-MPB equations}

Different from the IC-PB (i.e., steady PNP) equation, the IC-MPB equation cannot be solved analytically because of its strong nonlinearity. However, many kinds of numerical 
methods, which mainly belong to the relaxation method [11], are well known to solve this kind of nonlinear equation. Here, we use the simple Newton method only to examine that the IC-MPB equations provide the same solutions obtained by the MPNP equations. Namely, from Eq. (20), we define the residual function of the descretized potential $\phi_{i}\left(0 \leq \phi_{i} \leq v_{0}\right.$, $i=0,1, \ldots, n)$ for a fixed $c_{b}$ value at the position $x_{i}\left(0 \leq x_{i} \leq 1, i=0,1, \ldots, n\right)$ as

$$
R_{c_{b}}\left(\phi_{i}\right)=\frac{\phi_{i-1}+\phi_{i+1}-2 \phi_{i}}{\Delta x^{2}}-\frac{c_{b} \kappa^{2} \sinh \phi_{i}}{1+c_{b} \nu \cosh \phi_{i}}
$$

where $\Delta x=1 / n, n$ being the element number, and we use the approximation $\nabla^{2} \phi_{i} \simeq$ $\frac{\phi_{i-1}+\phi_{i+1}-2 \phi_{i}}{\Delta x^{2}}$. The derivative is

$$
R_{c_{b}}^{\prime}\left(\phi_{i}\right)=-\frac{2}{\Delta x^{2}}-\frac{c_{b} \kappa^{2}\left(\nu^{2}+\cosh \phi_{i}\right)}{\left(1+c_{b} \nu \cosh \phi_{i}\right)^{2}}
$$

Thus, by the Newton method, we obtain the fixed solution of the $s+1$ step as

$$
\phi_{i}^{(s+1)}=\phi_{i}^{(s)}-\omega R_{c_{b}}\left(\phi_{i}^{(s)}\right) / R_{c_{b}}^{\prime}\left(\phi_{i}^{(s)}\right),
$$

where $\omega=0.5$ is the relaxation parameter. By using the boundary conditions $\phi_{n}=v_{0}$ and $\phi_{0}=0$ at $x_{n}=1$ and $x_{0}=0$, respectively, we obtain the solution of Eq. (20) for a fixed $c_{b}$ value. Note that we judge the convergence by the condition $R_{c_{B}}\left(\phi_{i}\right)<10^{-2}$ for all $i$ values. Furthermore, from Eq. (21), we define the residual function of $c_{b}$ concerning the ion-conserving condition as

$$
I_{R}\left(c_{b}\right)=\int_{0}^{1} \frac{c_{b} \cosh \phi}{1+c_{b} \cosh \phi} d x-1 .
$$

Fortunately, since $I_{R}\left(c_{b}\right)$ is a monotonically increasing function of $c_{b}$, we obtain the solution that satisfies $I_{R}\left(c_{b}\right)=0$ (i.e., the solution of the IC-MPB equations) by the bisection method. Note that we judge the convergence by the condition $I_{R}\left(c_{B}\right)<10^{-3}$ as a first step.

\section{F. Finite-element finite-volume (FE-FV) method for solving the MPNP equations}

To examine the equivalence between the IC-MPB and MPNP equations numerically, we perform numerical calculations of the nonsteady MPNP equations [4] by the finite-element finite-volume (FE-FV) method because the ion-conserving condition is ensured by the continuous equation in the nonsteady formulation, as mentioned previously. The FE-FV method calculates the modified Nernst-Planck (MNP) equation part by the FV method, which conserves the ion numbers effectively, and calculates the Poison equation part by FEM, which 
can calculate the potential effectively (see e.g., Appendix A in Ref. [12]). That is, we calculate the nonsteady MPNP equations

$$
\begin{array}{r}
\frac{\partial c_{ \pm}}{\partial \tau}=\nabla_{n}\left[\nabla_{n} c_{ \pm} \pm c_{ \pm} \nabla_{n} \phi+\frac{\nu c_{ \pm}}{\left[2-\nu\left(c_{+}+c_{-}\right)\right]} \nabla_{n}\left(c_{+}+c_{-}\right)\right] . \\
2 \nabla_{n}^{2} \phi=-\kappa^{2} \rho_{n}
\end{array}
$$

where $\tau=t / T_{0}, T_{0}=W_{0}^{2} / D, \kappa=W_{0} / \lambda_{D}, \rho_{n}=c_{+}-c_{-}, D$ (typically $10^{-9} \mathrm{~m}^{2} / \mathrm{s}$ for water) is the ion diffusivity, $\lambda_{D}$ is the Debye screening length, and $t$ is time.

\section{RESULTS}

To clarify the meaning of the IC-MPB theory, we firstly show the results of the solution group of Eq. (9); i.e., Fig. 2 shows the calculation results of Eq. (9) at $C_{b} / C_{0}=1,10^{-10}$, and $10^{-15}$ under the condition that $\kappa=10, \nu=0.01$, and $v_{0}=V_{0} / \Phi_{c}=40$. Here, we confirmed that the solutions of $C_{b} / C_{0}=1$ and $10^{-10}$ do not satisfy Eq. (10), whereas the solution of $C_{b} / C_{0}=10^{-15}$ satisfies Eq. (10). Thus, by considering the argument of Sec. II-F, the solutions of Eq. (9) at $C_{b} / C_{0}=1$ and $10^{-15}$ correspond to the solutions of the MPB and IC-MPB solutions, respectively; thus, we denote "(mpb)" and "(icmpb)" for $C_{b} / C_{0}=1$ and $10^{-15}$ in Fig. 2, although they are calculated from Eq. (9) by the same method using the Newton method, which is the same method of Sec. II-E except that it provides the value of $C_{b}$ as a parameter. As shown in Fig. 2(a), the thickness of the condensation layer due to the steric effect decreases as $C_{b} / C_{0}$ decreases; the physical picture of the MPB theory is much different from that of the IC-MPB theory because the value of $C_{b} / C_{0}$ is completely different by the order of 15. Moreover, as shown in Fig. 2(b), the potential distribution of the IC-MPB theory is also very different from that of the MPB theory; i.e., the bulk electric field is screened at $C_{b} / C_{0}=1$, whereas it is not screened at $C_{b} / C_{0}=10^{-15}$. Obviously, the screened phenomenon is an artificial one in a confined space owing to the unphysical assumption of the MPB equation.

Figure 3 shows the comparison between the IC-MPB (lines) and MPNP (symbols) solutions for the dependences of $C_{-}$and $\phi$ on $X$ at $\kappa=10$. As shown in Fig. 3, they are in good agreement. In particular, the dependences of $C_{-}$on $X$ at $v_{0}=V_{0} / \Phi_{c}=80$ and 40 for various $\nu$ values are in good agreement between the IC-MPB and MPNP solutions, as shown in Figs. 3(a) and 3(c), although the dependences of $\phi$ on $X$ are slightly different, as shown 


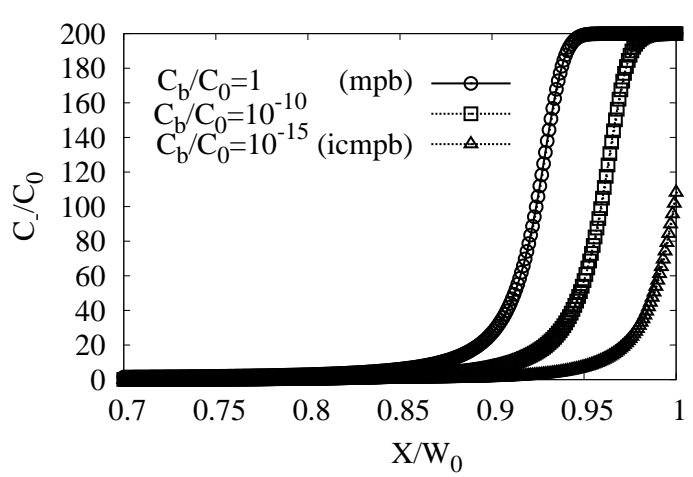

(a) Dependence of $C_{-}$on $X$.

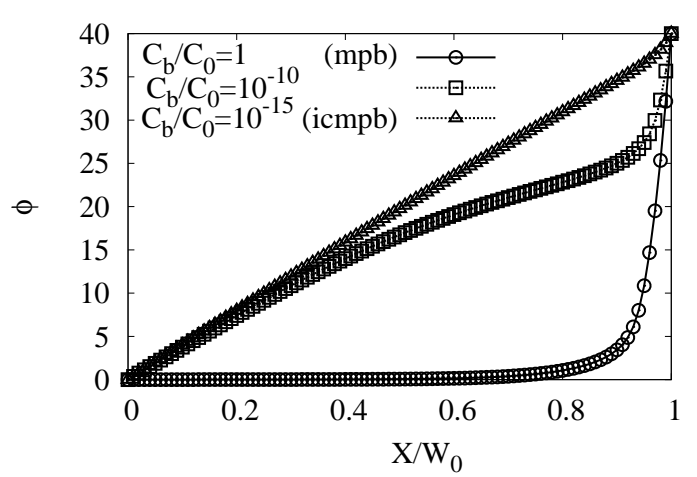

(b) Dependence of $\phi$ on $X$.

FIG. 2: Solution group of the general PB equation due to the difference in $C_{b} / C_{0}$. Here, $\kappa=10$, $\nu=0.01$, and $v_{0}=V_{0} / \Phi_{c}=40$.

in Figs. 3(b) and 3(d). This is because the numerical calculation of the nonsteady MPNP equations is slightly more difficult than that of the IC-MPB equations and thus the accuracy of the nonsteady MPNP calculations is slightly lower than that of the IC-MPB calculations. For example, for the MPNP and IC-MPB equations, the non-dimensional bulk concentration $C_{b} / C_{0}$ often becomes smaller than $10^{-15}(\ll 1)$ at high applied voltages, whereas the non-dimensional surface concentration reaches $2 / \nu(\gg 1)$; in this case, the accuracy of the numerical calculations for the nonsteady MPNP equations decreases.

\section{DISCUSSION}

We have clarified that the MPB solutions are surprisingly much different from the MPNP solutions in a confined space at high voltages (> $25 \mathrm{mV})$; however, by adding an ionconserving condition, the equations become equivalent to the MPNP equations. Thus, the argument using the MPB equation should be replaced by the more precise argument using the IC-MPB equations. Although the IC-MPB equations are equivalent to the steady MPNP equations, it is more useful for the argument of the steady phenomena because the calculation cost is intrinsically much lower than that of the nonsteady MPNP equations. Moreover, the relaxation method for the IC-MPB equation can be easily extended to the $2 \mathrm{D}$ and $3 \mathrm{D}$ problems. Furthermore, although there has been no way of examining the accuracy of the MPNP calculations at high applied voltages so far, our study provides the examination 


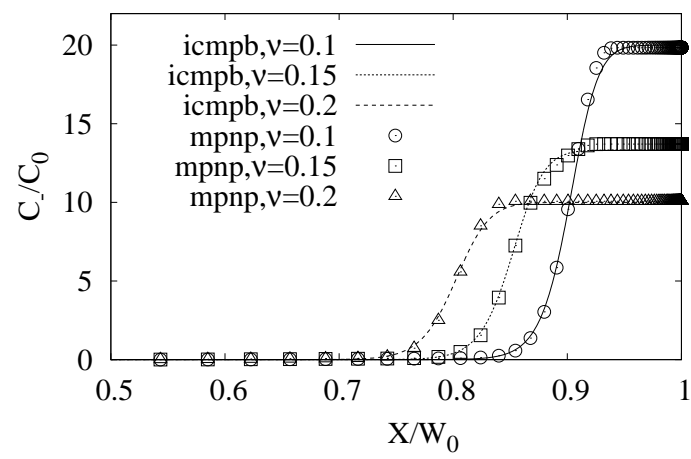

(a) Dependence of $C_{-}$on $X\left(v_{0}=80\right)$.

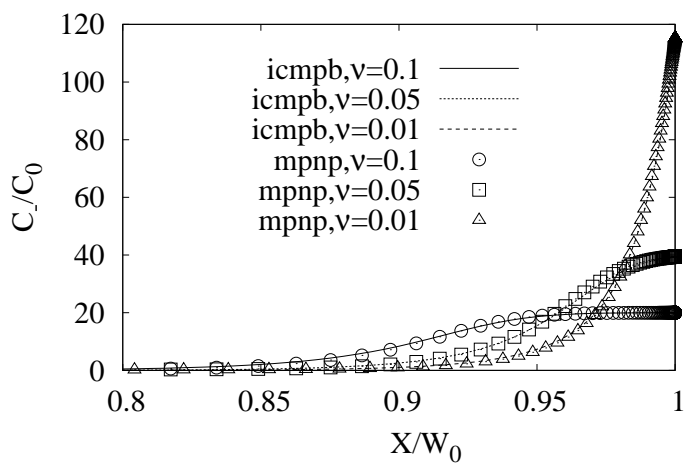

(c) Dependence of $C_{-}$on $X\left(v_{0}=40\right)$.

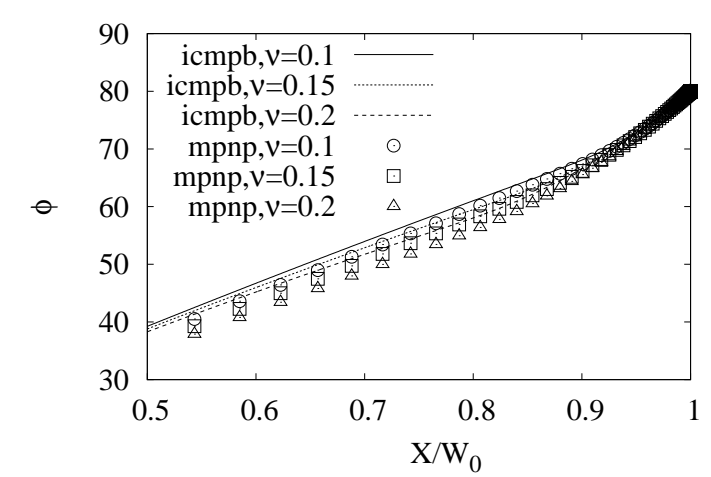

(b) Dependence of $\phi$ on $X\left(v_{0}=80\right)$.

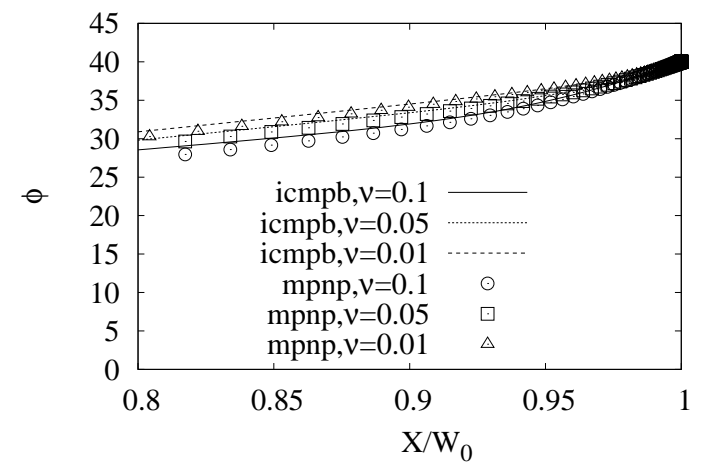

(d) Dependence of $\phi$ on $X\left(v_{0}=40\right)$.

FIG. 3: Comparison between the IC-MPB (lines) and MPNP (symbols) solutions. Here, $\kappa=10$.

method. Thus, our finding is useful for better understanding of the surface science between a fluid and a solid. In addition, our finding provides new insight into the design principle of various microfluidic devices using electro-osmosis phenomena in a confined space [10, 13]; e.g., although the experimental data (including flow reversal phenomena) of an ac electroosmotic (ACEO) pump [14] is analyzed using the results of the MPB equations [15], it should be reconsidered using the knowledge of the IC-MPB equations more precisely in the future. Furthermore, the experimental flow velocity of ACEO [or more generally induced charge electro-osmosis (ICEO)] is often much lower than the prediction of the standard theory and it is usually explained by ion crowding phenomena [5] suggested from the MPB theory. However, the IC-MPB theory [especially Fig. 2(a)] shows that ion crowding phenomena are much more unlikely to occur than the prediction of the MPB theory. Thus, we may need to reconsider this problem from the viewpoint of the IC-MPB theory. Moreover, as pointed out by Zhao [2], the conventional PNP model cannot explain the low-frequency 
dispersion amplitude and the characteristic frequency of particles quantitatively [16, 17]. Thus, our finding should be considered to correct the discrepancy between the theory and the experiment in the future.

\section{CONCLUSIONS}

On the basis of an improved free energy formulation considering a steric effect with an ion-conserving condition, we have successfully derived the ion-conserving modified PoissonBoltzmann (IC-MPB) equations that are equivalent to the steady modified Poisson-NernstPlanck (MPNP) equations that consider a steric effect of ions. Furthermore, we numerically examined the equivalence between the IC-MPB and MPNP equations at high applied voltages by comparing the IC-MPB solutions obtained by the Newton method with the MPNP solutions obtained by the finite-element finite-volume method. Surprisingly, the MPB solutions are much different from the MPNP (IC-MPB) solutions, which provide a correct answer, in a confined space, and thus we need to use the MPNP (i.e., IC-MPB) solutions. We believe that our findings contribute greatly to the surface science.

\section{Acknowledgments}

This work was partially supported by JSPS KAKENHI Grant Number JP16K05650.

[1] I. Borukhov, D. Andelman, and H. Orland, Phys. Rev. Lett. 79, 435 (1997).

[2] H. Zhao, J. Phys. Chem. C 114, 8389 (2010).

[3] M. S. Kilic, M. Z. Bazant, and A. Ajdari, Phys. Rev. E 75, 021502 (2007).

[4] M. S. Kilic, M. Z. Bazant, and A. Ajdari, Phys. Rev. E 75, 021503 (2007).

[5] M. Z. Bazant, M. S. Kilic, B. D. Storey, and A. Ajdari, Adv. Colloid Interf. Sci. 152, 48 (2009).

[6] G. Guoy, J. Phys. 9, 45 (1910).

[7] D. L. Chapman, Philos. Mag. 25, 475 (1913).

[8] D. Grahame, J. American Chemical Society 76, 4819 (1954).

[9] H. Sugioka, Phys. Rev. E 86, 016318 (2012). 
[10] H. Sugioka, Advances in Colloid and Interface Science 226, 44 (2015).

[11] A. Nicholls and B. Honig, j. Comp. Chem. 12, 435 (1991).

[12] H. Sugioka, Phys. Rev. E 90, 013007 (2014).

[13] M. Z. Bazant and T. M. Squires, Phys. Rev. Lett. 92, 066101 (2004).

[14] J. P. Urbanski, T. Thorsen, J. A. Levian, and M. Z. Bazant, Appl. Phys. Lett. 89, 143508 (2006).

[15] B. Storey, L. R. Edwards, M. S. Kilic, and M. Z. Bazant, Phys. Rev. E 77, 036317 (2008).

[16] D. Myers and D. Saville, Journal of Colloid and Interface Science 131, 461 (1989).

[17] L. A. Rosen and D. A. Saville, Langmuir 7, 36 (1991). 